Recepción: 08 / 09 / 2016

Aceptación: 30 / 01 / 2017

Publicación: 21 / 06 / 2017

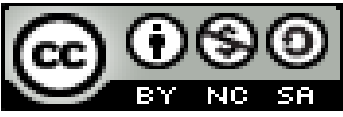

Ciencias de la educación

Artículo de Investigación

\title{
Algunas nociones acerca de las competencias en el idioma Inglés
}

\author{
Some notions about competences in the English language
}

\section{Algumas noções sobre as habilidades em Inglês}

\author{
Washington G. Mancero-Orozco ${ }^{\mathrm{I}}$ \\ wmancero@espoch.edu.ec \\ Luís A. Veloz-Andrade " \\ lveloz@espoch.edu.ec
}

Correspondencia: wmancero@espoch.edu.ec

\begin{abstract}
Magister en Administración para el Desarrollo Educativo, Licenciado en Ciencias de la Educación Profesor de Enseñanza Media en la Especialización de Idiomas: inglés y francés, Docente de la Escuela Politécnica de Chimborazo ESPOCH, Riobamba, Ecuador.

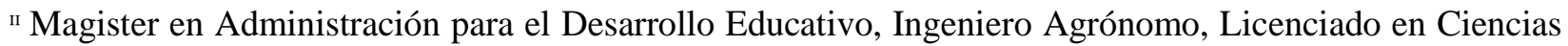
de la Educación Profesor de Enseñanza Media en la Especialización de Idiomas: inglés y francés, Docente de la Escuela Politécnica de Chimborazo ESPOCH, Riobamba, Ecuador.
\end{abstract}




\section{Resumen}

El concepto de competencia es de uso frecuente en la enseñanza de las lenguas, tanto nativas como extranjeras. Se busca desarrollar orgánicamente cuatro competencias lingüísticas básicas: hablar, escribir, escuchar y leer. El idioma inglés constituye una lengua franca y su dominio una habilidad de primer orden. A tales efectos se llevó a cabo una revisión bibliográfica la cual hace referencias acerca de las competencias en el idioma ingles (definición, competencia comunicativa, el aprendizaje de la escritura, entre otros) y se enunció algunos resultados de investigaciones relacionadas con la temática.

Palabras clave: enseñanza; competencias; inglés.

\section{Abstract}

The concept of competence is often used in the teaching of languages, both native and foreign. It seeks to organically develop four basic linguistic skills: speaking, writing, listening and reading. The English language is a lingua franca and its domain is a skill of the first order. To that end, a bibliographic review was carried out which makes references about the competences in the English language (definition, communicative competence, learning of writing, among others) and some results of research related to the subject.

Keywords: teaching; competences; english.

\section{Resumo}

O conceito de concorrência é frequentemente usado em ensino de línguas, tanto nativa e estrangeira. Procura desenvolver organicamente quatro competências linguísticas básicas: fala, escrita, audição e leitura. O idioma Inglês é uma língua franca uma habilidade e domínio de primeira ordem. Para este efeito, foi realizada uma revisão da literatura que faz referências sobre habilidades no idioma Inglês (definição, competência comunicativa, aprendizagem, escrita, etc.) e alguns resultados de pesquisas relacionadas ao tema enunciado.

Palavras chave: ensinar;competência; inglês.

\section{Introducción}

Entre las muchas revoluciones que ha vivido la humanidad, las que han cambiado de forma radical el modo de pensar, y que, a su vez, han logrado transformar la sociedad donde los seres humanos se desenvuelven, son las que han contado con el conocimiento como principal actor. 
(Valdés Linares, M T, González Valdés, S, Díaz Cabeza, I, Verdayes Vives, A, Díaz Lobo, L M. 2010).

El conocimiento ha adquirido un poder que irrumpe en todas las esferas de la vida y demanda el desarrollo de capacidades para el procesamiento, reestructuración y recreación crítica de la acelerada difusión de la información. (López M E, Tello A M.2008).

Valdés Linares, M, et al. (2010), plantea que el nuevo papel del conocimiento está induciendo transformaciones profundas en la educación, por lo que este se convierte en un factor clave para poner en marcha los procesos necesarios para enfrentar los desafíos del mundo actual. Es amplio el reconocimiento por parte de instituciones como la UNESCO sobre los avances de la educación tanto primaria, secundaria como superior y otras instituciones y organizaciones académicas, científicas y profesionales, que mediante sus funciones de enseñanza, formación e investigación y servicios, representan un factor necesario para el desarrollo social.

El conocimiento y dominio de una lengua extranjera es una herramienta fundamental que se exige como parte de las habilidades profesionales de un individuo. Además, la incursión de las nuevas tecnologías de la comunicación y la información ha revolucionado el estilo de vida del ser humano. Estas herramientas tecnológicas permiten establecer contacto con personas en cualquier lugar del mundo, estudiar a distancia, ampliar los conocimientos en diversas áreas del saber, conocimientos y saberes que cambian y se amplían a cada segundo.

\section{Desarrollo}

En la actualidad, la lengua inglesa ha tenido mayor importancia debido a las necesidades que plantea el siglo XXI, en la que los estudiantes requieren tener un dominio del mismo. (Orientaciones para la enseñanza de inglés en el Bachillerato general. 2010)

La idea de enseñar Inglés es dar la posibilidad a las y los estudiantes de comunicarse en una segunda lengua, con personas dentro y fuera del país; brindarles herramientas necesarias para el conocimiento y el desarrollo de las cuatro habilidades lingüísticas básicas (escucha, habla, lectura y escritura), así como una serie de sub-habilidades, cuyo objetivo primordial es el dominio del idioma. En esta línea, el aprendizaje del idioma Inglés debe desarrollarse en contexto y las actividades deben proveer oportunidades para que al desarrollar un tema central, se logren 
integrar las habilidades por medio de actividades reales. (Orientaciones para la enseñanza de inglés en el Bachillerato general. 2010)

El concepto de competencia es de uso frecuente en la enseñanza de las lenguas, tanto nativas como extranjeras. Se busca desarrollar orgánicamente cuatro competencias linguiísticas básicas: hablar, escribir, escuchar y leer. (Escalante López M. 2004).

En igual forma una competencia permite identificar, seleccionar, coordinar y movilizar de manera articulada e interrelacionada un conjunto de saberes diversos en el marco de una situación educativa en un contexto específico. (Enfoque centrado en competencias. 2012)

Los pilares de la educación y campos formativos relacionados con las competencias son: Aprender a conocer, en la adquisición de una amplia cultura; aprender a hacer, en acciones y competencias para poder enfrentar situaciones diversas; aprender a ser, construirse internamente capacidades cognitivas, afectivas y rectoras del individuo; y aprender a convivir, comprensión entre los seres humanos. Tolerancia y respeto a los seres humanos. (Castañeda Rodríguez C, Gayosso- Poza Rica D M. 2012).

Con referencia a lo anterior, para los investigadores y docentes en Lenguas Extranjeras (LE) el término "competencia" se ha convertido en un término bastante familiar, ya que se menciona en repetidas oportunidades dentro del objetivo general o específico en la mayoría de los programas de las asignaturas. No obstante, son pocos los que realmente confirman hasta dónde esa competencia, bien sea comunicativa, gramatical, discursiva o cualquier otra se ha logrado al final del curso. Si partimos de la afirmación de que en cuanto a lenguaje, la competencia es "una aptitud para saber y saber-hacer que se actualiza o no según la intencionalidad del sujeto que habla o interpreta" (Pulido Barrios R, Muñoz O. 2012), la competencia discursiva pasa a ser la que podrá dar cuenta de ese saber y saber-hacer.

La enseñanza del inglés en los momentos actuales ha tenido un gran auge, siendo el idioma que se utiliza mundialmente. No obstante, aún no se ha logrado el nivel idóneo de competencia comunicativa en las habilidades que entraña el dominio de la lengua extranjera: expresión oral y escrita, comprensión oral y lectora. (Alonso Avila M 2014). 
Hoy en día, el propio desarrollo científico - técnico precisa de una constante preparación y actualización de nuestros profesionales donde la búsqueda de información y la comprensión de lo que se investiga deriva en un posterior trabajo de redacción final que conlleva a que se realice de forma eficiente. Por lo que todo lo anteriormente expuesto exige de un encomiable trabajo en aras de preparar a nuestros educandos en el desarrollo de la habilidad de hablar, escribir, escuchar y leer. (Alonso Ávila M. 2014)

\section{Competencia comunicativa en el idioma inglés}

La competencia comunicativa se manifiesta como una suma de subcompetencias o dimensiones; no obstante, se debe tener en cuenta que, sean unas u otras, estas tienen que integrarse de forma simultánea. (Berenguer-Román I, Roca-Revilla M. Torres-Berenguer M. 2016)

Alcón (citado por Berenguer-Román I, et al 2016) considera que la competencia comunicativa está compuesta por la interrelación de subcompetencias tales como: la competencia discursiva, que incluye componentes linguísticos, textuales y pragmáticos, respecto a la cual opina que está influenciada por las habilidades lectoras, auditivas, orales y de escritura; la competencia lingüística, la cual incluye al conocimiento gramatical y a todos los aspectos del sistema lingüístico; las competencias textual y pragmática, cuyos componentes son necesarios para la construcción e interpretación del discurso, y, por último, la competencia estratégica, que incluye a la comunicación y a las estrategias de aprendizaje.

Las competencias lingüísticas o comunicativas en hablantes no nativos en idioma inglés no están homogéneamente instaladas. Las personas evidencian diferentes capacidades para producir enunciados que respeten las reglas gramaticales de una lengua en términos de vocabulario, sintaxis, semántica y pronunciación adecuada; elementos necesarios no sólo para darse a entender en dicho idioma, sino también para comprender sus enunciados.( Rodríguez Garcés, Carlos. 2015).

Cabe agregar, quienes se dedican a la enseñanza/aprendizaje de lengua extranjera esperan de sus estudiantes que sepan hacer uso de las formas gramaticales y del vocabulario que han aprendido a lo largo de sus estudios, poniéndolos en práctica para comunicar sus conocimientos, experiencias y emociones, de forma coherente y cohesiva en diversas situaciones reales de comunicación.( Pulido Barrios R, Muñoz O. 2012). 
Para el desarrollo de la competencia comunicativa es imprescindible que conozcan la cultura de ese país, sus reglas sociales, sus costumbres.

\section{La escritura en el aprendizaje del idioma inglés}

La escritura tiene un lugar primordial dentro de la comunicación, pues en dependencia de la situación, en ocasiones, es más factible utilizar la escritura que la expresión oral. Esta habilidad tiene una importancia específica dentro del propio proceso de enseñanza- aprendizaje del idioma ya que sirva de instrumento a este.

Con independencia de las motivaciones para el aprendizaje de las lenguas extranjeras entre los estudiantes, se han identificado ciertos elementos que, como aportes a dicho aprendizaje, presenta la escritura: Eleva el nivel de conciencia sobre los procesos lingüísticos que ocurren en la lengua materna en sentido general y de su escritura en lo particular. Ayuda en el proceso del aprendizaje de la lengua extranjera al ser la escritura un canal más para su proceso de interiorización. Contribuye al desarrollo de las habilidades del pensamiento y a la creatividad. Incrementa la capacidad para la planificación, organización y control de la actividad verbal. Permite la asimilación, por parte del aprendiz, de las formas de comunicación escritas de otras culturas y acercarse a ellas desde un punto de vista crítico, con respeto a las formas de expresión de otros hombres y de este modo contribuir individualmente a la paz entre los pueblos. Potencia las posibilidades de elevar el nivel cultural en lo general y educativo en particular y con ello, un mejor acceso al mercado del trabajo. Incrementa las posibilidades para la inserción del individuo en una nueva comunidad discursiva y defender su identidad, cultura e ideología. (Alonso Ávila M 2014).

En estos momentos, las publicaciones con mayor difusión en el mundo se realizan en idioma inglés, todas las radios y televisoras internacionales tienen programación en inglés, las actividades culturales, políticas, sociales y científicas de orden internacional incluyen el inglés como una de las lenguas del evento. Sea en papel o soporte electrónico, la mayoría de los libros o artículos en publicaciones periódicas o resúmenes de libros sobre temas científicos, tecnológicos o sociales, se publican en inglés o, al menos, exigen un resumen en idioma inglés, y esto mismo sucede con los manuales para productos electrodomésticos, alimentos y las comunicaciones 
telefónicas, por satélite o correo electrónico, que incluyen las comunicaciones personales, listas de discusión de todo tipo y tema, noticias y otros. (Alonso Ávila M 2014).

Saborit Leiva, Raiza Texidor, Portuondo Ferrer (2014) en su investigación concluyen que la enseñanza de la escritura debe ser estimulada desde el nivel elemental de competencia comunicativa con la finalidad de desarrollar una buena redacción en los estudiantes. Se sugiere que el profesor retome o incluya entre sus estrategias de enseñanza la escritura como proceso y reflexione sobre la importancia de su uso en el aula. Se recomienda, además, enfocar más este tema tan intrínsecamente relacionado con el logro de la competencia comunicativa.

Según Rico Yate, Ramírez Montoya, Montiel Bautista (2016), los hallazgos de su investigación indican que la competencia oral de los estudiantes en un curso de inglés en b-learning se beneficiaron con el uso de los recursos educativos abiertos al usarse estrategias didácticas con tareas significativas, ya que se abren las posibilidades de extensión de la práctica del idioma en la interacción y producción, y se promueve el enfoque comunicativo.

Estudio realizado por Sanhueza Jara, Burdiles Fernández (2012), el cual se propuso establecer algunos puntos de encuentro entre la descripción de la competencia comunicativa en inglés de un grupo de escolares chilenos y el perfil de estrategias de aprendizaje de lengua extranjera que les caracteriza. El grupo de estudiantes observado en este estudio se caracterizó por manifestar niveles deficientes en varios otros aspectos de la competencia comunicativa, tales como la comprensión lectora y auditiva, la producción escrita y el uso del idioma. El bajo nivel de competencia comunicativa demostrado en uso del idioma puede significar carencias en aspectos gramaticales. A su vez, la tendencia a un nivel pobre de competencia en producción escrita guarda relación con deficiencias en uso del idioma, donde se evidencia un disminuido conocimiento del sistema de la lengua. Los problemas de comprensión, en tanto, se manifiestan igualmente en relación con los registros orales y los escritos.

En su investigación Concepción Pacheco A, Díaz Vázquez E (2006), la enseñanza del idioma inglés en las Ciencias Biomédicas, no incide favorablemente en la toma de decisiones individuales y colectivas, de los estudiantes, con relación al desarrollo de hábitos, habilidades y relaciones sociales de los mismos, combinando aspectos propios de la organización eficiente de la enseñanza: óptima participación de los estudiantes, dinamismo y entretenimiento. Los 
estudiantes al tratar de interpretar roles, no obtienen resultados favorables en relación con el desarrollo de su competencia comunicativa en idioma inglés.

Para concluir, las competencias implican transformar la didáctica tradicional a una conformada y desarrollada en los cuatro pilares de la educación para así diseñar estrategias didácticas y llevarlas a cabo a través de técnicas en las cuales sus propósitos y objetivos estén basados en las necesidades de los alumnos y sobre todo en contextos reales de acuerdo a su nivel educativo.

\section{Referencias bibliográficas}

Alonso Ávila M (2014). Idioma inglés, escritura y desarrollo: un reto para el profesional de la Cultura Física en la Facultad de Guantánamo. Recuperado de http://www.efdeportes.com/efd189/idioma-ingles-escritura-y-desarrollo.htm

Berenguer-Román I, Roca-Revilla M, Torres-Berenguer M. (2016). La competencia comunicativa en la enseñanza de idiomas. Revista Dom. Cien., 2(2), pp. 25-31.

Concepción Pacheco A, Díaz Vázquez E (2006). La competencia comunicativa y su relación con la enseñanza del idioma inglés en las "Ciencias Biomédicas". Gaceta Médica Espirituana 2006; 8(3). Consultado el 8 de julio de 2016. Recuperado de http://bvs.sld.cu/revistas/gme/pub/vol.8.\%283\%29_07/p7.html

Castañeda Rodríguez C, Gayosso-Poza Rica D M. (2012). Importancia del Desarrollo de competencias en la adquisición del idioma Inglés. Consultado el 8 de julio de 2016. Recuperado de https://www.uv.mx/.../files/.../Pon_CinthyaC_DianaC_CICoatzacoalcos_CIPozaRica.p.

Escalante López M (2004). Un modelo de desarrollo de competencias en la enseñanza del idioma inglés. Investigación educativa 8(14). Consultado el 8 de julio de 2016. Recuperado de http://revistasinvestigacion.unmsm.edu.pe/index.php/educa/article/view/7094

Enfoque centrado en competencias. (2012). Consultado el 8 de julio de 2016. Recuperado de http://www.dgespe.sep.gob.mx/reforma_curricular/planes/lepri/plan_de_estudios/enfoque_centra do_competencias

López ME, Tello AM. (2008). Las lenguas extranjeras en carreras de la Facultad de Ciencias Humanas de la Universidad Nacional de San Luis (Argentina). Fundamentos en Humanidades IX 
(1): 1743-70. Consultado el 27 de agosto de 2016. Recuperado de http://redalyc.uaemex.mx/src/inicio/ArtPdfRed.jsp?iCve $=18417103$

Orientaciones para la enseñanza de inglés en el Bachillerato general. (2010). Consultado el 28 de julio de 2016. Recuperado de: www.dgb.sep.gob.mx/informacion-academica/.../orientacionesenzenanza-ingles.pdfl.

Pulido Barrios R, Muñoz O. (2012). La competencia discursiva y el texto oral en lengua extranjera: un estudio de caso. Consultado el 13 de agosto de 2016. Recuperado de https://dialnet.unirioja.es/descarga/articulo/3979278.pdf

Rodríguez Garcés, Carlos. (2015). Competencias comunicativas en idioma inglés: La influencia de la gestión escolar y del nivel socioeconómico en el nivel de logro educativo en L2-inglés.

Perfiles educativos, 37(149), 74-93. Consultado el 28 de julio de 2016. Recuperado de http://www.scielo.org.mx/scielo.php?script=sci_arttext\&pid=S0185-

26982015000300005\&lng=es\&tlng=es.

Rico Yate, J P, Ramírez Montoya, M S, \& Montiel Bautista, S. (2016). Desarrollo de la competencia oral del inglés mediante recursos educativos abiertos. Apertura (Guadalajara, Jal.), 8(1), 00006. Consultado el 28 de julio de 2016. Recuperado de: http://www.scielo.org.mx/scielo.php?script=sci_arttext\&pid=S166561802016000200006\&lng=es\&tlng=es.

Saborit Leiva, Hildo, Texidor Pellón, Raiza, \& Portuondo Ferrer, Nereida. (2014). Sugerencias para desarrollar la escritura en las clases de inglés. Revista Habanera de Ciencias Médicas, 13(4), 605-611. Consultado el 28 de julio de 2017. Recuperado de http://scielo.sld.cu/scielo.php?script=sci_arttext\&pid=S1729$519 X 2014000400012 \& \operatorname{lng}=\mathrm{es} \& \ln g=\mathrm{es}$.

Sanhueza Jara, María Gabriela, Burdiles Fernández, Gina América. (2012). Diagnóstico de la competencia comunicativa en inglés de un grupo de escolares chilenos: puntos de encuentro con su perfil estratégico. Folios, (36), 97-113. Consultado el 13 de agosto de 2016. Recuperado de http://www.scielo.org.co/scielo.php?script=sci_arttext\&pid=S0123$48702012000200006 \& \operatorname{lng}=\mathrm{en} \& \operatorname{tn} \mathrm{ln}=\mathrm{es}$ 
Valdés Linares, María Teresa, González Valdés, Sisely, Díaz Cabeza, Idania, Verdayes Vives, Aniuska, \& Díaz Lobo, Luis Manuel. (2010). La enseñanza del inglés en las ciencias médicas: su repercusión social. Revista de Ciencias Médicas de Pinar del Río, 14(3), 26-31. Consultado el 13 de agosto de 2016. Recuperado de http://scielo.sld.cu/scielo.php?script=sci_arttext\&pid=S1561$31942010000300006 \& \operatorname{lng}=\mathrm{es} \& \operatorname{tn} g=\mathrm{es}$ 